\title{
Implementation of an IP Telephony System Based on Asterisk PBX; A Case Study of Garden City University College, Ghana
}

\author{
Siddique Abubakr Muntaka \\ Kessben University College \\ School of Physical Sciences \\ Kuntanase-Kumasi, Ghana
}

\author{
Faiza Hussein \\ Garden City University College \\ Computer Science Department \\ Kenyasi-Kumasi, Ghana
}

\author{
Paul Sarfo \\ Garden City University College \\ Computer Science Department \\ Kenyasi-Kumasi, Ghana
}

\begin{abstract}
Garden City University is a higher learning institution in Ghana, in the second capital, Kumasi. The institution has several faculties and departments. The communication requirement of the institution has changed over years after establishment, ranging from traditional communication delivery using office messengers to the use of mixed mode where they still deliver messages through messengers or call using GSM/telephone networks, which is usually costly as compared to other available methods. Voice over internet protocol is a prevailing technology that can enhance an organizations communication. Asterisk PBX is a software based private business exchange that has the capability to outsmart a traditional analogue PBX system. This research project therefore uses the power vested in the open source asterisk PBX to deploy a prototype of a communication system for Garden city university college, using open source liblinphone library and Software development kit to create a softphone for both inbound and outbound communication among staff and the university administration. The system uses Raspberry pi for the prototype. The same project can be replicated on a powerful computer architecture for relatively higher capacity of communication. Evaluation of the system is done to ascertain information about the project. The evaluation of the prototype helps in building similar system for production use. A GSM modem with voice support is later used to interface the Asterisk PBX network with public switch telephone network so that staff could dial regular telephone numbers.
\end{abstract}

\section{Keywords}

Communication, Private Business Extension (PBX), Opensource, liblinphone, Raspberry pi, GSM, PSTN.

\section{INTRODUCTION}

Internet Telephony uses Voice over Internet Protocol (VoIP) and it is one of the developing technology that has the ability to provide low cost and yet a high quality and availability of service. VOIP technology support multimedia data transmission such as audio and video. In general, VOIP system involves sending voice information in digital form in discrete packets rather than in the traditional circuit committed protocols of the public switched telephone network (PSTN).

A major advantage derived from VoIP and Internet telephony is that it avoids the tolls charged by ordinary telephone service. When an Internet protocol telephone calls are initiate, there is a conversion of the analogue voice signal to digital format and compression or translation of the signal into IP packets for transmission over a network or the Internet; the process is therefore reversed at the receiving end [1]. The term VOIP can also be used to refer to computer hardware, software, and computer network systems, that perform functions that are traditionally performed by telephone equipment.

Asterisk is a software version of a telephone private branch exchange (PBX) that allows telephones to link with a variety of hardware technologies to make calls to one another, and to connect to telephony services such as public switched telephone network (PSTN) and voice over Internet Protocol (VoIP) services. The name comes from the asterisk symbol

Asterisk was created in 1999 by Mark Spencer of Digium [2] This communication server software was originally designed for Linux based operating systems. Asterisk can run on a variety of operating systems that includes NetBSD, OpenBSD, FreeBSD, MacOS, and Solaris, and can also be installed on an embedded system. Because Asterisk PBX is open source and Linux based system, it presents a lot of potentials that could be configured to extend it use. It however needs command over the knowledge in Linux to effectively extend it use to maximize the full potentials.

\section{LITERATURE REVIEW}

The literature review helps relate the study to a larger scope of ongoing discourse in academics. Thus, filling in gaps in the literature and extending earlier studies. This chapter therefore presents a well-organized critical appreciation of related and relevant literature that are conceptually integrated within the logic of the proposed project. This section of the research seeks to delve into past or previous works that relates to Asterisk PBX communication systems setup, techniques, and the related technologies. The researchers will also discuss voice over internet protocol and internet telephony. The analysis of the existing knowledge related to the subject of inquiry brings good representation and critical review of the existing literature.

In cost-quality based consumer perception analysis of voice over internet protocol, Douskalis [1] explained that VoIP (voice over IP) is voice delivered using network and Internet protocol. This term is used in IP telephony for a set of facilities for managing the delivery of voice information using the Internet protocol (IP). In general, this means sending voice information in digital form using discrete packets rather than the traditional circuit committed protocols of the public switched telephone network (PSTN). In VoIP and Internet telephony, tolls charges by ordinary telephone service providers can be avoided. The steps involved in originating an Internet telephone call is the conversion of the analogue voice 
signal to digital format and compression or translation of the signal into IP packets for transmission over the Internet or over a network. The process is reversed at the receiving end. Douskalis research indicates the cost effectiveness and networking strength of VoIP as compared to traditional telephone system. Quality of service in terms of latency is however a generic problem in voice over internet protocol projects. These problems may not be too different with systems like 3cx and freepbx which are all projects based on Asterisk PBX; an open source communication server based on Linux or Unix operating systems.

In defining asterisk pbx, Meggelen [3] states that asterisk is a software form of the hardware PBX and it runs mainly on the open source Linux operating systems. It was created by Mark Spencer and helped along the way by Jim Dixon. Like every other open source project, Asterisk PBX gained massive contribution and help by an enthusiastic open source community. asterisk is now at the heart of many sophisticated corporate phone systems [4]. Asterisk was born out of frustration at the high costs to buying very basic telephone system for Mark Spencers Linux support business.

The use of voice over internet protocol (VoIP) services has seen substantial improvement and growth over this recent years [5]. There is more awareness of the benefits that VoIP offers looking at cost and ubiquity. Many corporate institutions and residential users are now familiar with this kind of technology. Between 2008 and 2013, Netherlands and France recorded 32 and 27 percent respectively for VoIP communications. VoIP calls in the European Union represented 8 percent of the traffic as of December 2007 [6]

There are commercial VoIP options but many are expensive systems that run old and complicated code on obsolete hardware. Asterisk PBX software runs on Linux and can interoperate with almost all standards-based telephony equipment or systems.

Under a research conducted on the performance evaluation of Asterisk PBX, Sousa [7] uses a network analysis tool called Wireshark to take assessment of the performance of the PBX. Sousa's research tried to describe Asterisk PBX in detail, giving instructions, configuration, installation and testing for a result of the systems performance. Sousa states that currently PBX (Private Branch exchange) are an almost indispensable tool in the enterprise world. PBX allow employees to make connections among the internal telephones of an enterprise and connect them to the public switched telephone network (PSTN). With the increased use of Internet today, there is a need to understand what services are offered, how to configure and evaluate the performance of a PBX with support for voice over IP (VoIP). Asterisk PBX is a cost free and open source software for Linux, which supports multiple protocols such as SIP, SCCP, H.323, IAX2, etc.

Though VoIP promises low cost and quality communication, it also comes with some obvious disadvantages [8]. According to Graham \& Ure [9], VoIP in its various forms raises several complex questions that goes beyond the proximity of whether consumers get cheaper telephone calls, but at the same time IP technology is ushering in a world of peer to peer communications that provides both opportunities and dangers for businesses. The authors in this paper considered the various challenges raised by Voice over Internet Protocol (VoIP) and states that it includes commercial, technological, marketing and regulatory issues. This was done by first exploring the technological issues such as quality assurance and load control, then marketing issues by contrasting the various service providers and their approaches, and finally regulatory concerns by comparing European and US approaches to issues such as emergency services, numbering, law enforcement and security. The authors findings proved the deficiencies that could come with VoIP technology aside it positive capabilities as compared to traditional telephone system.

Session Initiation Protocol (SIP) is a signaling protocol used in the setup of any type of session. SIP call control uses Session Description Protocol (SDP) to describe the call details such as being audio or video, a shared application, codec type, and the size of packets. In the use of SIP, a Universal Resource Locator (URI) handles the identification of a logical destination.

\subsection{Review of Related Architecture}

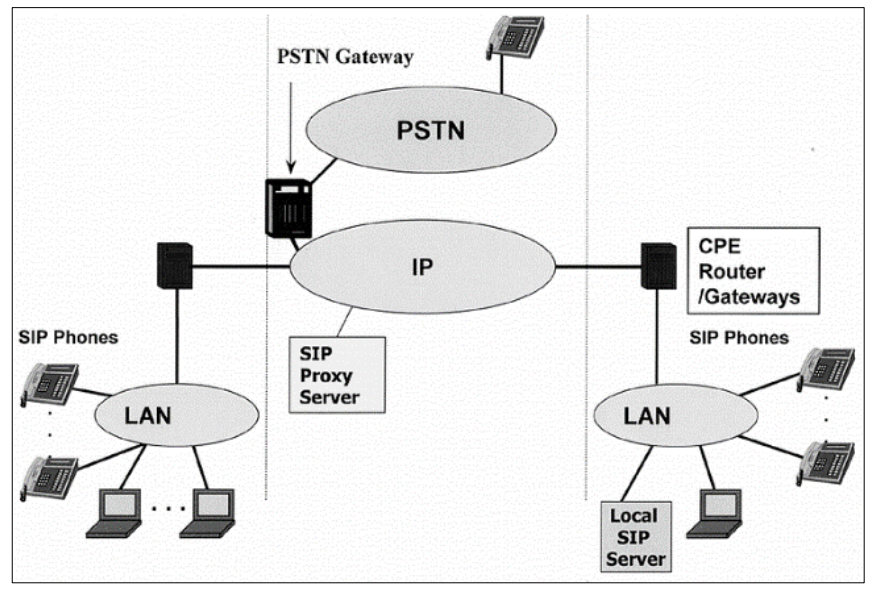

Fig 1: End -to-End VoIP [10]

Figure 1 is a diagram of an IP telephone system connected to a wide area network through a gateway. The IP phones are connected to the Local Area Network. Voice calls can be made locally over the Local Area Network. The IP phones include codec's that are digitize by encoding and decoding the speech. The IP phones can also help packetize and depacketize the encoded speech into IP packets. Calls between different geological locations can be made over the wide area network. Connections to the PSTN can be established through VoIP gateways.

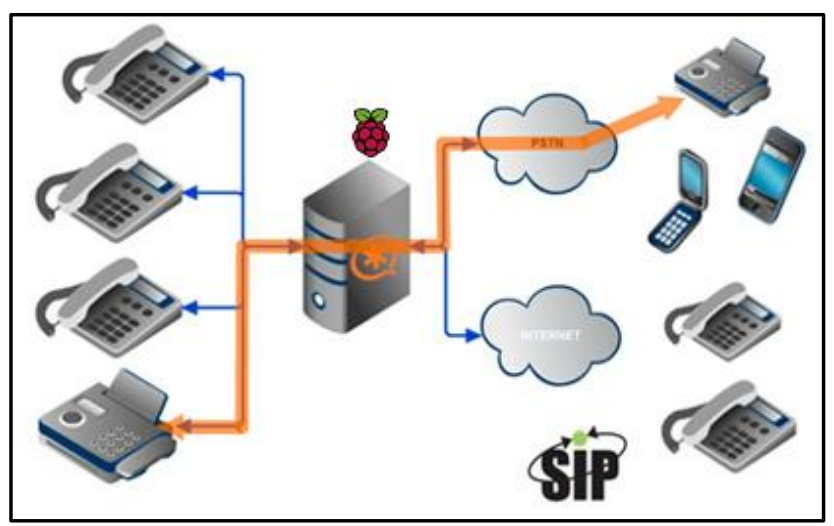

Fig 2: Freepbx, Asterisk using raspberry pi [11]

Figure 2 shows a server that runs Asterisk Freepbx and has Session Initiation protocol ability. Client systems like smart phones or computers can be used to connect to the server to enable the users to communicate with each other over the same network. An example of such phone systems are Cisco 
Unified SIP Phone 3900 series.

\section{METHODOLOGY - DESIGN AND PROTOTYPE}

Design methodology was implemented by using system development life cycle. A prototype was used to setup the Asterisk PBX server that serves as the engine of the whole project. The prototype developed served as a sample of the project and it is tested to feel how it will look like in a production environment.

\subsection{Development tools and Platform}

The design of a system is largely affected by the performance and output it is expected to deliver. Consequently, elements that encompass hardware reliability and software processing are the determinants of the efficiency of a system. This provides the foundation for software and systems development. Efficiency, performance and maintainability are highly dependent on the design of the system. The following were the tools and softwares used in the project;

- $\quad$ Asterisk PBX; FreePBX \& Raspbx (for raspberry pi)

- $\quad$ Raspberry pi 3 model B

- $\quad$ Micro SD card as storage Disk for Raspberry Pi

- USB cable for power

- Wireless router

- Network cables

- $\quad$ Putty for remote control

- Win32Disk Imager

- $\quad$ Open source Liblinphone Library and SDK

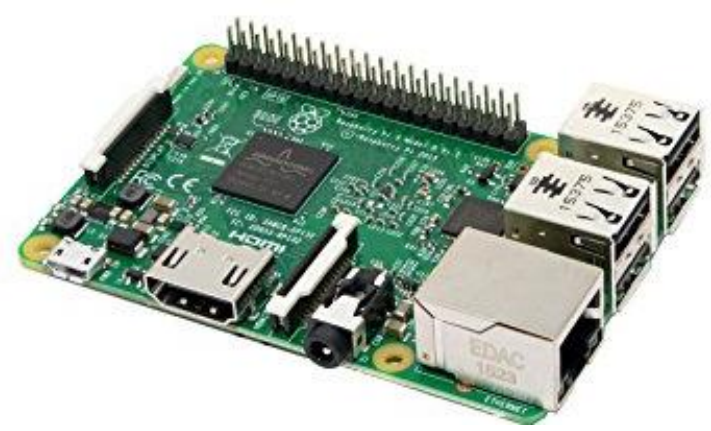

Fig 3: Raspberry Pi Board

Figure 3 is a single-board computer which was developed in the United Kingdom by the Raspberry Pi Foundation to promote teaching of basic computer science in schools and in developing countries. It is small, like the size of a credit card. The original model became far more popular than anticipated, selling outside the target market for uses such as robotics. Raspberry pi does not include peripherals such as keyboards, mouse and cases. However, some accessories have been included in several official and unofficial designs.

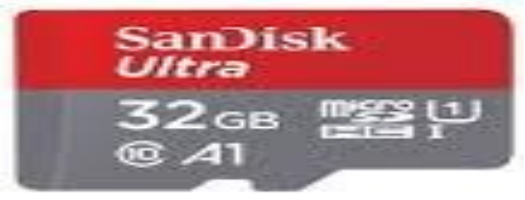

Fig 4: Micro SD Card
Figure 4 is a microSD which is a type of removable flash memory card used for storing information. SD is an abbreviation that stands for Secure Digital. MicroSD cards are sometimes referred to as $\mu \mathrm{SD}$ or $\mathrm{uSD}$. This is usually used in mobile phones and other devices like Raspberry Pi, Banana $\mathrm{Pi}$, Pine A64 and other single board computers or embedded systems.

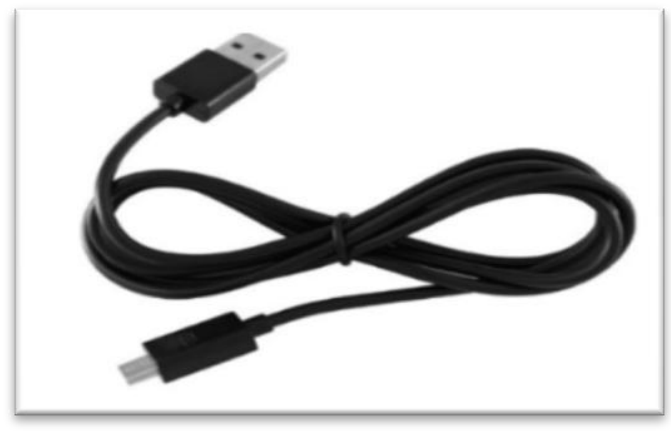

Fig 5: Mini USB Cable

Figure 5 is USB which stands for Universal Serial Bus. There are four wires inside the USB. Usually, red, green, white, and black cord, with each of these wires having a corresponding code: Red colour indicates the positive wire with 5 volts of DC power. Black is the ground wire in almost all electronic devices.

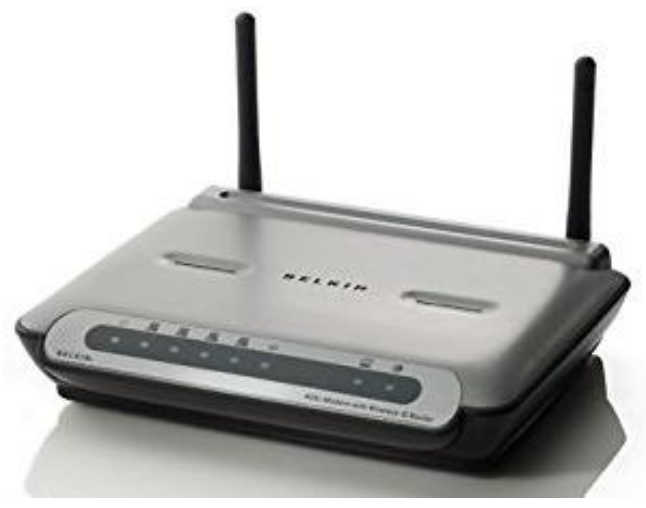

Fig 6: Wireless Router

Figure 6 is a wireless router and it is an electronic device that performs the functions of routing and it may include the functions of a wireless access point. It is used to provide access to Internet or a private computer network. Depending on the manufacturer and model, it can function in a wired local area network with few port for LAN communication. It has support for wireless-only LAN, or mixed, wired and wireless network. The main purpose is to create a network.

\subsection{System Architectural Design}

The architecture of the system under development is divided into three main parts. Namely the Server architecture, the network architecture and the soft phone architecture. Server architecture shows how the server is designed for various system components to connect and communicate. The network architecture also shows the connection medium between users on the other side that uses the soft phone and the server. The soft phone architecture has layout of how the soft phone is design and the possible features. 


\subsection{Server Architecture / Design}

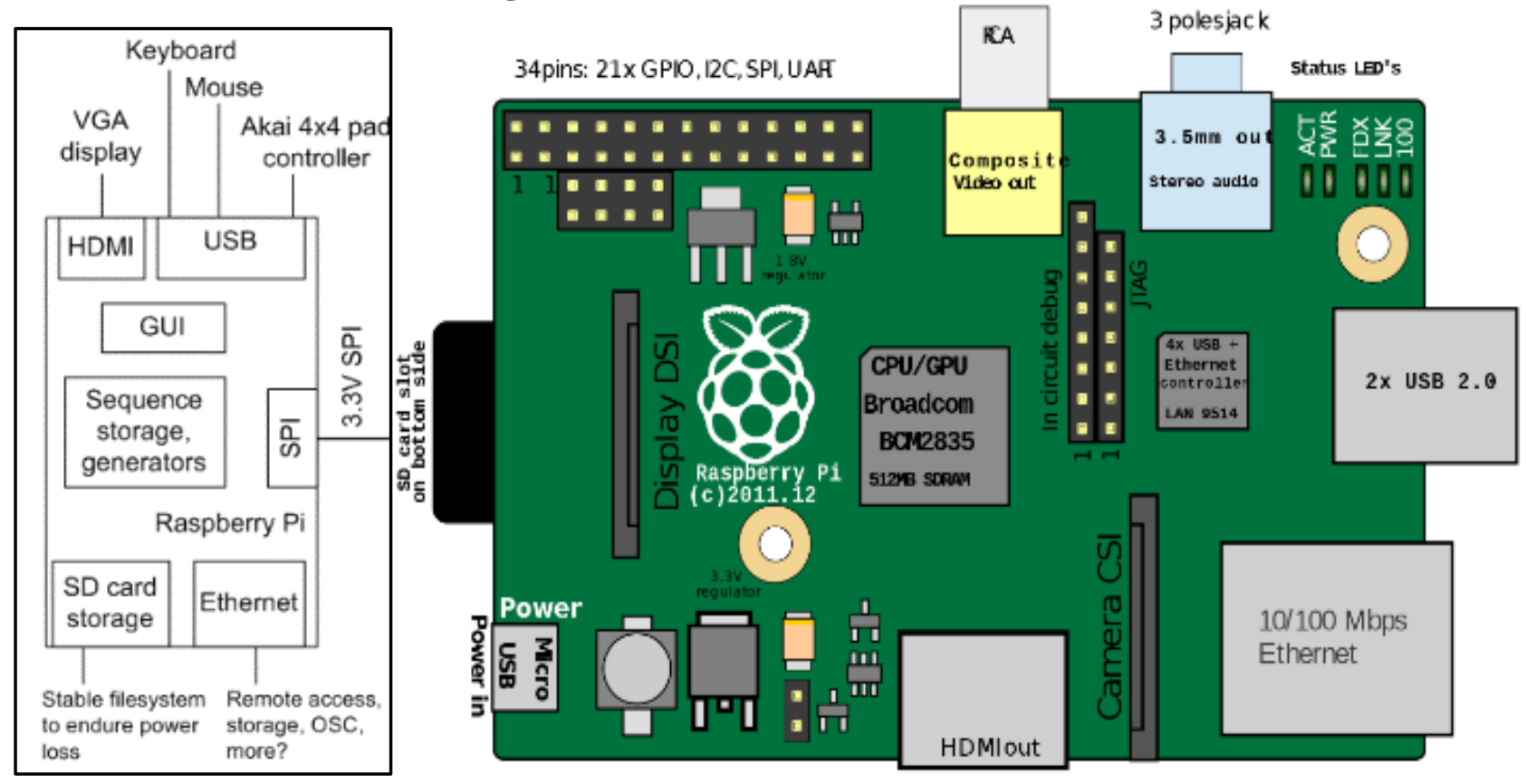

Fig 7: Single Board Computer; Raspberry Pi

In figure 7 above, the single board computer (SBC) used is Raspberry Pi 3 Model B. This system has different integrated or embedded components including USB, audio jack, mini usb, wireless, etc.

\subsection{Network Architecture / Design}

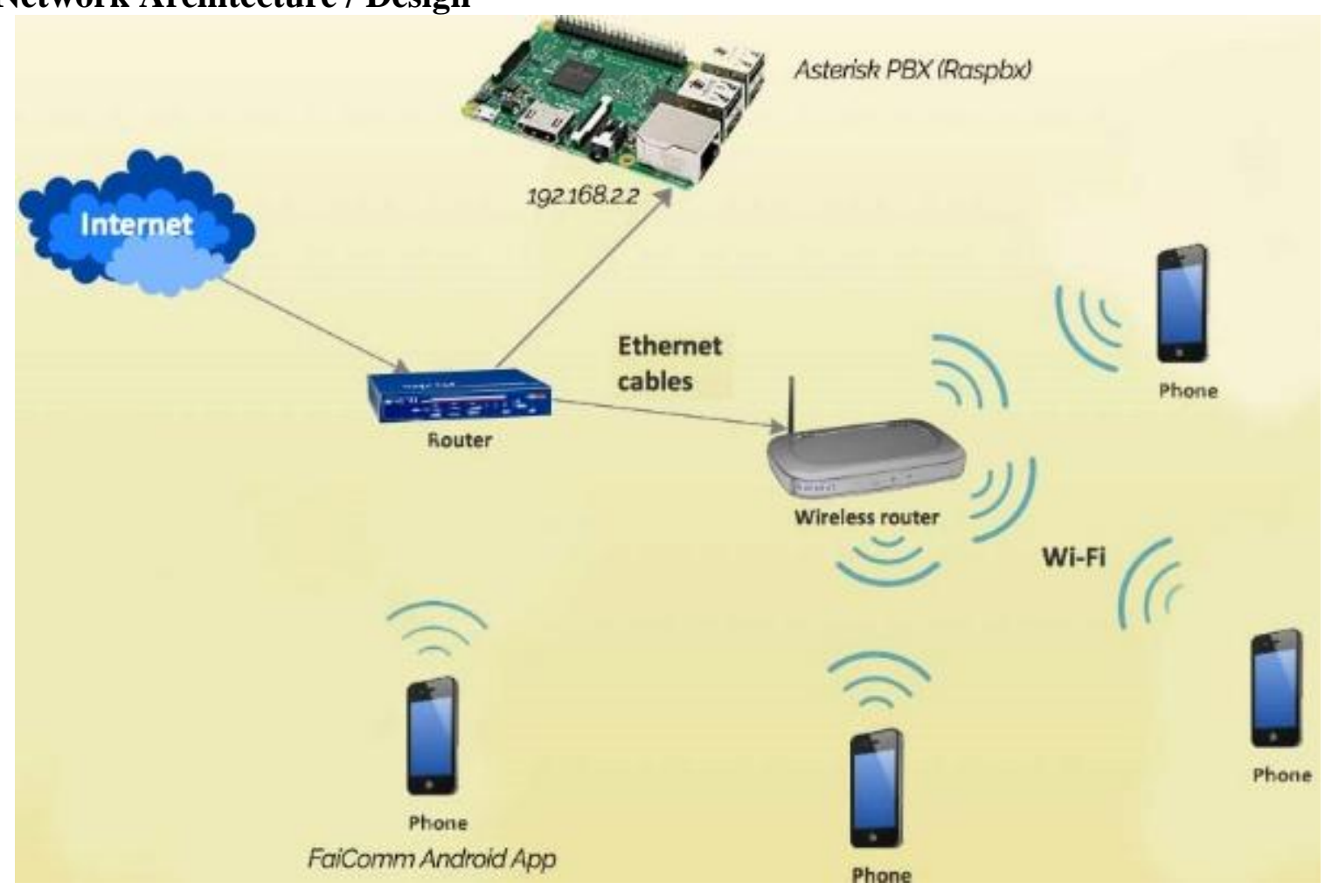

Fig 8: Network Architecture

Figure 8 defines the layout of how the network is implement with both the communication server and the soft phone deployed. The server and the application must both be on the same network as displayed using wireless network devices or access points. 


\subsection{Soft phone Architecture / Design}

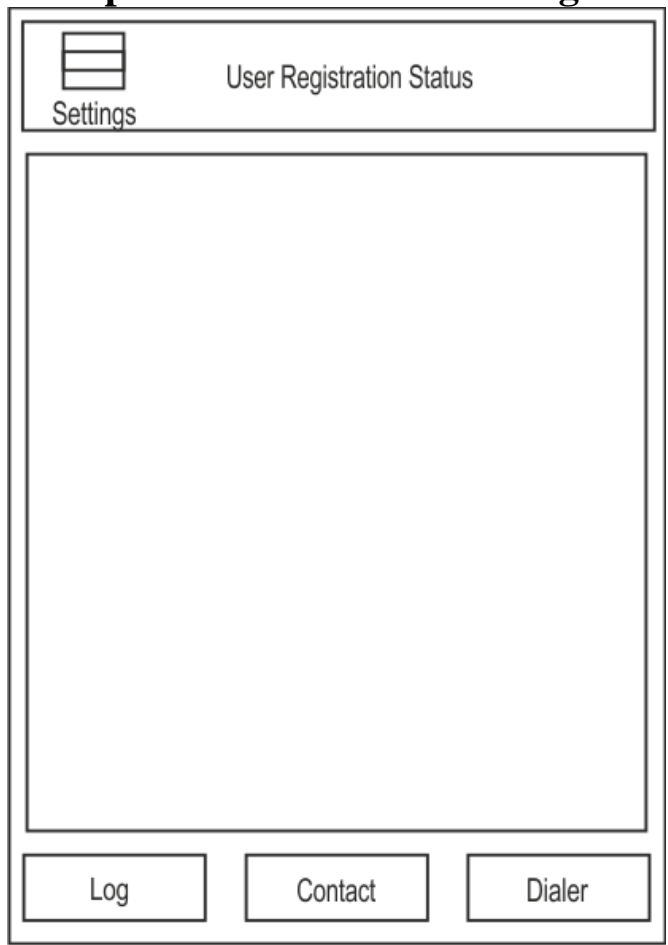

Fig 9: Soft phone.

The above image in figure 9 shows the user interface design and layout of the android app that serves as a client application connecting each users phone to the server.

\subsection{Server setup}

Depending on the host system, Asterisk PBX is downloaded based on different flavours. FreePBX can be downloaded and installed on a 32 bit or 64bit system. The raspberry pi has a package called RasPBX which can be downloaded from raspberry-asterisk.org. Win32Disk Imager is a software tool used to write the Raspbx image onto a micro SD card. Alternatively, after acquiring a raspberry pi with Raspbian Os on the Sd Card already installed. Login to the terminal of the system and download Asterisk PBX via commands, as well as FreePBX, Apache, MySQL, installed by commands one after the other.

\subsection{Database Design}

Below are tables that represent the databases for setting up the communication server. Thus, table 1 to 4 .

Table 1: Database for extension numbers. This keeps records of the various numbers.

\begin{tabular}{|c|}
\hline Extension number \\
\hline User extension \\
Display name \\
Outbound CID \\
Secret \\
\\
\hline
\end{tabular}

Table 2: Database for users. This keeps records of the management on users of various extension lines or numbers

\begin{tabular}{|c|}
\hline User manager \\
\hline Select User Directory: \\
Link to a Default User \\
Username \\
Password for New User \\
Group \\
\hline
\end{tabular}

Table 3: Database for users. This keeps records of the users of various extension lines or numbers

\begin{tabular}{|c|}
\hline User Table \\
\hline Login Name \\
Description \\
Password \\
Groups \\
Primary Linked Extension \\
\hline
\end{tabular}

Table 4: Database for users to keep records of the users details

\begin{tabular}{|c|}
\hline User details \\
\hline First Name \\
Last Name \\
Display Name \\
Title \\
Company \\
Department \\
Email Address \\
Cell Phone Number \\
Work Phone Number \\
Home Phone Number \\
Fax Phone Number
\end{tabular}




\subsection{User Interface Design: Server}

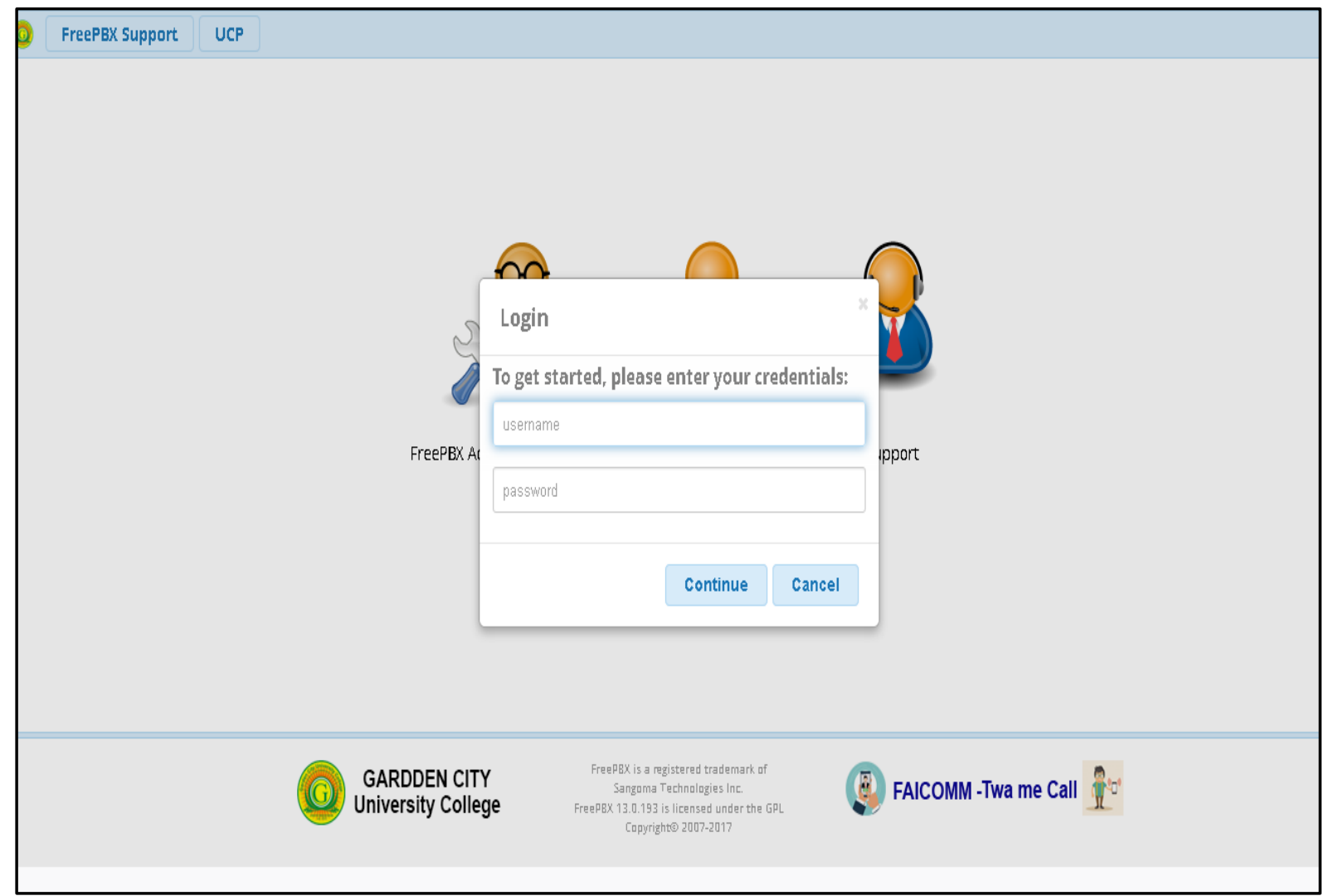

Fig 10: Administrator login.

Figure 10 shows the login page for the administrator of the server through a web browser. The IP Address or the domain name of the server is entered on a browser to launch the first page for authentication into the server..

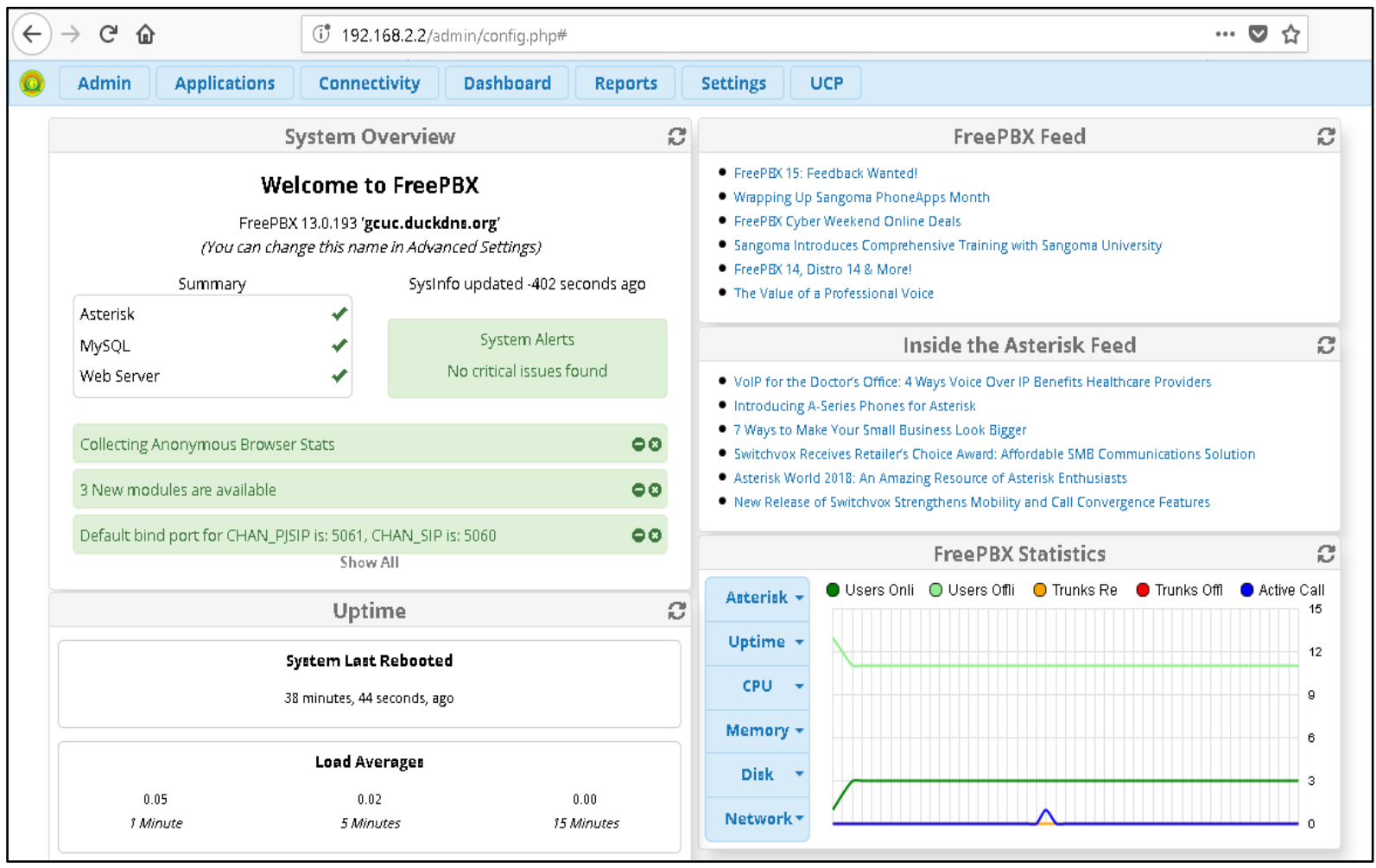

Fig 11: Dashboard of administrator login. 
Figure 11 is the main landing page when the administrator of the communication server logs in to manage or configure the

\subsection{Interface Design: Softphone}

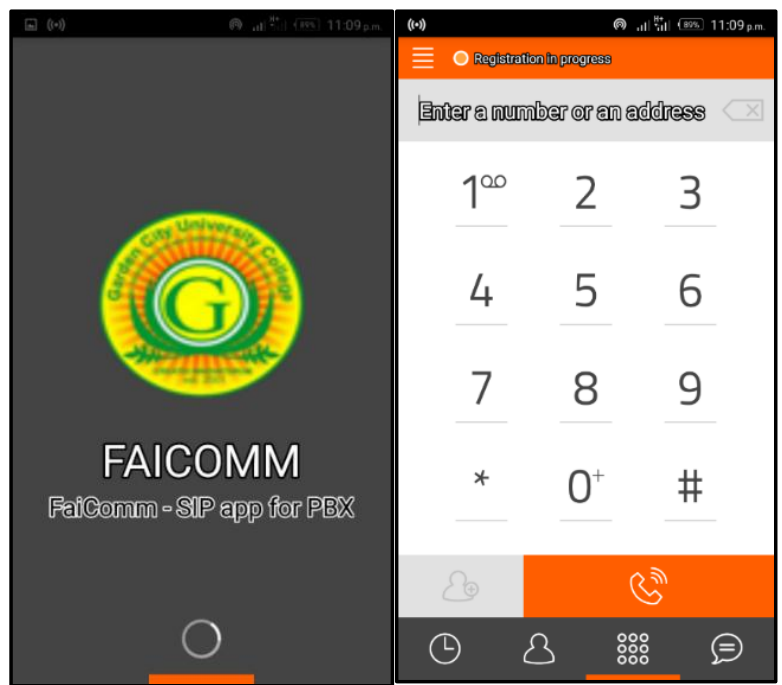

Fig 12: Softphone user Interface.

Figure 12 show the interface and look of android software phone application when launched to connect to the PBX.

\section{RESULTS AND CONCLUSION}

The research project aimed at creating a communication system to enhance campus communication. Testing the system reveals that communication over the wireless network was successful with relatively some appreciable level of delay as the number of client connected to the Asterisk PBX Server increases. The delay was understandable since the prototype used Raspberry pi which comes with a limited computing resources. The call quality was however very good. The researchers further enhanced the setup by integrating the system with public switch telephone networks in Ghana like Vodafone, MTN, Tigo, Airtel, etc. This was made successful using a Huawei USB Modern. The research project was very challenging especially because the system is Linux based. The result was satisfying since the main objectives was realized. system

\section{REFERENCES}

[1] Douskalis, B. (1999). IP Telephony: The Integration of Robust VolP Services, Prentice Hall, ISBN 978-0-13014118-7, New Jersey.

[2] Digium. (2017). Asterisk® Features. Retrieved June, 2017, from http://www.asterisk.org/features

[3] Van Meggelen, J., Madsen, L., \& Smith, J. (2007). Asterisk: The future of telephony. "O'Reilly Media, Inc.".

[4] Charny, B. (2017). Is the telephone industry ready for open source? Retrieved September 2017, from http://news.com/ Is +the+ telephone+ industry+ready+ for+ open+source/20081082_35737703.html part= rss\&tag $=5737703 \&$ subj $=$ news

[5] Schneir, J. R., \& Plückebaum, T. (2010). VoIP network architectures and impacts on costing. Info, 12(3), 59-72. https://doi.org/10.1108/14636691011040486

[6] Comission of the European Communities (2009), Progress Report on the Single European Electronic Communications Market (14th Report), SEC(2009)376/2, Volume 1, Part 2, Commission of the European Communities, Brussels.

[7] Sousa, L. (2001) Performance evaluation of the Asterisk PBX.https://pdfs.semanticscholar.org/8c3e/6a80b943a85 bad2f959c9562f02f7068cb57.pdf

[8] Walsh, T.J. and Kuhn, D.R. (2005), "Challenges in securing voice over IP", IEEE Security \& Privacy, Vol. 3 No. 3, pp. 44-49.

[9] Graham, T., \& Ure, J. (2005). IP telephony and voice over broadband. Info, 7, 8-20 https://doi.org/10.1108/14636690510607268

[10] Vaishav, C. (2006). Voice over Internet Protocol ( VoIP): The Dynamics of Technology and Regulation.

[11] Buyapi.ca (2017) Free PBX (Asterisk). Retrieved September 2017, from https://www.buyapi.ca/free-pbxasterisk/ 